IJMMS 27:1 (2001) 27-37

PII. S0161171201005580

http://ijmms.hindawi.com

(C) Hindawi Publishing Corp.

\title{
QUATERNION CR-SUBMANIFOLDS OF A QUATERNION KAEHLER MANIFOLD
}

\author{
BASSIL J. PAPANTONIOU and M. HASAN SHAHID
}

(Received 7 August 2000)

\begin{abstract}
We study the quaternion CR-submanifolds of a quaternion Kaehler manifold. More specifically we study the properties of the canonical structures and the geometry of the canonical foliations by using the Bott connection and the index of a quaternion CR-submanifold.
\end{abstract}

2000 Mathematics Subject Classification. 53C20, 53C21, 53C25.

1. Introduction. The notion of a CR-submanifold of a Kaehler manifold was introduced by Bejancu [3]. Subsequently a number of authors studied these submanifolds (see [4] for details). In [1], Barros et al. studied quaternion CR-submanifolds of a quaternion Kaehler manifold and obtained many interesting results. The aim of this paper is to continue the study of quaternion CR-submanifolds of a quaternion Kaehler manifold. The paper is organized as follows: in Section 2 we collect some basic formulas and results for later use and in Section 3 we study some properties of canonical structures, particularly its parallelism and QR-product. In Section 4 we study the geometry of the canonical foliations using the Bott connection and the index of a quaternion CRsubmanifold. Finally, as an extension of the work of Chen [5] for the Kaehler manifolds we give a complete classification of the totally umbilical quaternion CR-submanifolds of a quaternion Kaehler manifold.

2. Preliminaries. Let $\bar{M}$ be a quaternion Kaehler manifold with metric tensor $g$ and quaternion structure $V$ [7]. We will denote by $\psi_{1}=I, \psi_{2}=J$, and $\psi_{3}=K$ a local basis of almost Hermitian structures for $V$.

Let $X$ be a unit vector tangent to the quaternion Kaehler manifold $\bar{M}$. Then the vectors $X, I X, J X, K X$ form an orthonormal frame. Let $Q(X)$ be the quaternion section determined by $X$. Any plane in a quaternion section is called a quaternion plane and the sectional curvature of a quaternion plane is called a quaternion sectional curvature. A quaternion Kaehler manifold is called a quaternion space form, which is denoted by $\bar{M}(c)$, if its quaternion sectional curvature is equal to a constant $c$ at any point of the manifold. The curvature tensor $\bar{R}$ of $\bar{M}(c)$ is given by, [7],

$$
\begin{aligned}
\bar{R}(X, Y) Z=\frac{c}{4}[ & g(Y, Z) X-g(X, Z) Y+\sum_{r=1}^{3} g\left(\psi_{r} Y, Z\right) \psi_{r} X \\
& \left.-g\left(\psi_{r} X, Z\right) \psi_{r} Y+2 g\left(X, \psi_{r} Y\right) \psi_{r} Z\right],
\end{aligned}
$$

where $\psi_{1}=I, \psi_{2}=J, \psi_{3}=K$. 
Let $M$ be a Riemannian manifold isometrically immersed in a quaternion Kaehler manifold $\bar{M}$. We also denote by $g$ the metric tensor induced on $M$. If $\nabla$ is the covariant differentiation induced on $M$, the Gauss and Weingarten formulas are given by

$$
\bar{\nabla}_{X} Y=\nabla_{X} Y+h(X, Y), \quad \bar{\nabla}_{X} N=-A_{N} X+\nabla_{X}^{\perp} N
$$

respectively, for any $X, Y$ tangent to $M$ and $N$ normal to $M$. Here $h$ and $\nabla^{\perp}$ are the second fundamental form associated with $M$, and the connection of the normal bundle, respectively. The second fundamental tensor $A_{N}$ is related to $h$ by

$$
g\left(A_{N} X, Y\right)=g(h(X, Y), N)
$$

A differentiable distribution $D_{x}$ on $M$ such that $\psi_{r}\left(D_{x}\right) \subseteq D_{x}$ for all $r=1,2,3$ is called a quaternion distribution. In other words, $D_{x}$ is a quaternion distribution if $D_{x}$ is contained into itself by its quaternion structure.

It is known [1] that a submanifold $M$ of a quaternion Kaehler manifold $\bar{M}$ is called a quaternion CR-submanifold if it admits a quaternion distribution $D_{x}$ such that its orthogonal complementary distribution $D_{x}^{\perp}$, is totally real, that is, $\psi_{r}\left(D_{x}^{\perp}\right) \subseteq T_{x}^{\perp} M$ for all $x \in M$ and $r=1,2,3$, where $T_{x}^{\perp} M$ denotes the normal space of $M$ at $x$.

A submanifold $M$ of a quaternion Kaehler manifold $\bar{M}$ is called a quaternion (resp., totally real) submanifold if $\operatorname{dim} D_{x}^{\perp}=0$ (resp., $\operatorname{dim} D_{x}=0$ ). A quaternion CR-submanifold is said to be proper if it is neither quaternion nor totally real.

We denote by $\mu$ the subbundle of the normal bundle $T^{\perp} M$ which is the orthogonal complement of $\psi_{1} D^{\perp} \oplus \psi_{2} D^{\perp} \oplus \psi_{3} D^{\perp}$, that is,

$$
T^{\perp} M=\psi_{1} D^{\perp} \oplus \psi_{2} D^{\perp} \oplus \psi_{3} D^{\perp} \oplus \mu ; \quad g\left(\mu, \psi_{r} D^{\perp}\right)=0 .
$$

The mean curvature vector $H$ of $M$ in $\bar{M}$ is defined by $H=(1 / n)$ trace $h$, where $n$ denotes the dimension of $M$. If we have

$$
h(X, Y)=g(X, Y) H
$$

for any $X, Y \in T M$, then $M$ is called a totally umbilical submanifold. In particular, if $h(X, Y)=0$ identically for all $X, Y \in T M, M$ is called a totally geodesic submanifold. Finally $M$ is called mixed totally geodesic if $h(X, Y)=0$ for $X \in D, Y \in D^{\perp}$. For totally umbilical CR-submanifolds, equations (2.2) take the forms

$$
\bar{\nabla}_{X} Y=\nabla_{X} Y+g(X, Y) H, \quad \bar{\nabla}_{X} N=-g(H, N) X+\nabla_{X}^{\perp} N
$$

The Codazzi equation for a totally umbilical CR-submanifold $M$, is given by

$$
\bar{R}(X, Y ; Z, N)=\mathcal{g}(Y, Z) g\left(\nabla_{X}^{\perp} H, N\right)-\mathcal{g}(X, Z) g\left(\nabla_{Y}^{\perp} H, N\right) .
$$

DEFINITION 2.1 (see [1]). Let $M$ be a quaternion CR-submanifold of a quaternion Kaehler manifold $\bar{M}$. Then $M$ is called a QR-product, if $M$ is locally the Riemannian product of a quaternion submanifold and a totally real submanifold of $\bar{M}$. 
For any $X \in T M$ and $N \in T^{\perp} M$, we put

$$
\begin{aligned}
& \psi_{r} X=P_{r} X+Q_{r} X, \\
& \psi_{r} N=t_{r} N+f_{r} N,
\end{aligned}
$$

where $P_{r} X, t_{r} N$ (resp., $Q_{r} X, f_{r} N$ ) are the tangential (resp., the normal) components of $\psi_{r} X$ and $\psi_{r} N$ for $r=1,2,3$.

For the second fundamental form $h$, the covariant differentiation is defined by

$$
\left(\bar{\nabla}_{X} h\right)(Y, Z)=\nabla_{X}^{\frac{1}{X}} h(Y, Z)-h\left(\nabla_{X} Y, Z\right)-h\left(Y, \nabla_{X} Z\right)
$$

and the Gauss-Codazzi equations are given by

$$
\begin{gathered}
R(X, Y, Z, W)=\bar{R}(X, Y, Z, W)+g(h(X, W), h(Y, Z))-g(h(X, Z), h(Y, W)), \\
{[R(X, Y) Z]^{\perp}=\left(\bar{\nabla}_{X} h\right)(Y, Z)-\left(\bar{\nabla}_{Y} h\right)(X, Z), \quad \forall X, Y, Z, W \text { tangent to } \bar{M},}
\end{gathered}
$$

where $R$ is the curvature tensor associated with $\nabla$ and $\perp$ in (2.12) denotes the normal component.

We collect from Barros et al. [1] the following results which we shall need in the sequel.

LEMMA 2.2. Every quaternion submanifold of a quaternion Kaehler manifold is totally geodesic.

LEMMA 2.3. The quaternion distribution $D$ of a quaternion $C R$-submanifold $M$ in $a$ quaternion Kaehler manifold $\bar{M}$ is integrable if and only if $h(D, D)=0$.

LEMMA 2.4. Let M be a quaternion CR-submanifold of a quaternion Kaehler manifold $\bar{M}$. Then the leaf $M^{\perp}$ of $D^{\perp}$ is totally geodesic in $M$ if and only if $g\left(h\left(D, D^{\perp}\right), \psi_{r} D^{\perp}\right)=0$, $r=1,2,3$.

LEMMA 2.5. Let M be a quaternion CR-submanifold of a quaternion Kaehler manifold $\bar{M}$. Then

$$
A_{\psi_{r} W} Z=A_{\psi_{r} Z} W \quad \text { for any } W, Z \in D^{\perp}
$$

3. Canonical parallel structures and QR-product. Let $P_{r}, f_{r}, Q_{r}$, and $t_{r}$ be the endomorphisms and the vector-bundle-valued 1-forms defined in (2.8), respectively. We define the covariant differentiation of $P_{r}, Q_{r}, t_{r}$, and $f_{r}$ as follows:

$$
\begin{array}{ll}
\left(\bar{\nabla}_{X} P_{r}\right)(Y)=\nabla_{X}\left(P_{r} Y\right)-P_{r} \nabla_{X} Y, & \left(\bar{\nabla}_{X} Q_{r}\right)(Y)=\nabla_{X}^{\perp}\left(Q_{r} Y\right)-Q_{r} \nabla_{X} Y, \\
\left(\bar{\nabla}_{X} t_{r}\right)(N)=\nabla_{X}\left(t_{r} N\right)-t_{r} \nabla_{X}^{\perp} N, & \left(\bar{\nabla}_{X} f_{r}\right)(N)=\nabla_{X}^{\perp}\left(f_{r} N\right)-f_{r} \nabla_{X}^{\perp} N,
\end{array}
$$

for any vector fields $X, Y \in T M$ and $N \in T^{\perp} M$.

The endomorphisms $P_{r}$ (resp., the endomorphisms $f_{r}$, the 1-forms $Q_{r}$ and $t_{r}$ ) are parallel if $\bar{\nabla} P_{r}=0$ (resp., $\bar{\nabla} f_{r}=0, \bar{\nabla} Q_{r}=0$, and $\bar{\nabla} t_{r}=0$ ). 
Now using the definition of a quaternion Kaehler manifold and taking account of (2.2), (2.8), we can easily obtain the following:

$$
\begin{aligned}
\left(\bar{\nabla}_{X} P_{r}\right)(Y) & =A_{Q_{r} Y} X+t_{r} h(X, Y), \\
\left(\bar{\nabla}_{X} Q_{r}\right)(Y) & =f_{r} h(X, Y)-h\left(X, P_{r} Y\right), \\
\left(\bar{\nabla}_{X} t_{r}\right)(N) & =A_{f_{r} N} X-P_{r} A_{N} X, \\
\left(\bar{\nabla}_{X} f_{r}\right)(N) & =-h\left(X, t_{r} N\right),
\end{aligned}
$$

for any $X, Y \in T M$ and $N \in T^{\perp} M$.

REMARK 3.1. Since the second fundamental form is symmetric, it follows from (3.2) that $P_{r}$ is parallel if and only if

$$
A_{\psi_{r} U} V=A_{\psi_{r} V} U, \quad \forall U, V \in T M
$$

Now if we set $V=X \in D$ in (3.6), we find that $A_{\psi_{r} U} X=0$ for all $U \in T M$, which is equivalent to $g\left(h(X, Y), \psi_{r} U\right)=0$ for any $X \in D$, and $Y, U \in T M$. In particular $g\left(h(X, Y), \psi_{r} Z\right)=0$ for any $X \in D$ and $Y, Z \in D^{\perp}$.

Thus, using Lemma 2.4 we obtain the following lemma.

LEMMA 3.2. Let $M$ be a quaternion CR-submanifold of a quaternion Kaehler manifold $\bar{M}$. If $P_{r}$ is parallel then the leaf $M^{\perp}$ of $D^{\perp}$ is totally geodesic in $M$.

Now we state and prove the following proposition.

Proposition 3.3. Let $M$ be a quaternion CR-submanifold of a quaternion Kaehler manifold $\bar{M}$. Then $Q_{r}$ is parallel if and only if $t_{r}$ is parallel.

Proof. Suppose $t_{r}$ is parallel. Then from (3.4) we have

$$
A_{f_{r} N} U=P_{r} A_{N} U, \text { for any } U \in T M
$$

Thus for any vector fields $U, V \in T M$ and $N \in T^{\perp} M$, we get

$$
g\left(A_{f_{r} N} U, V\right)=g\left(P_{r} A_{N} U, V\right)
$$

or equivalently

$$
f_{r} h(U, V)-h\left(U, P_{r} V\right)=0,
$$

that is, $\bar{\nabla} Q_{r}=0$.

The proof of the converse statement is similar.

LEMMA 3.4. Let $M$ be a QR-product of a quaternion Kaehler manifold $\bar{M}$. Then

(a) $\nabla_{Z} X \in D$,

(b) $\nabla_{X} Z \in D^{\perp}$, for all $X \in D$ and $Z \in D^{\perp}$. 
Proof. By using (2.2) and the definition of a quaternion Kaehler manifold, we find

$$
\psi_{r} \nabla_{Z} X=\nabla_{Z} \psi_{r} X+h\left(Z, \psi_{r} X\right)-\psi_{r}(X, Z) \quad \text { for } X \in D, Z \in D^{\perp} .
$$

The above equation yields

$$
\begin{aligned}
g\left(\psi_{r} \nabla_{Z} X, \psi_{r} W\right) & =g\left(\nabla_{Z} \psi_{r} X, \psi_{r} W\right)+g\left(h\left(Z, \psi_{r} X\right), \psi_{r} W\right), \\
g\left(\nabla_{Z} X, W\right) & =g\left(h\left(Z, \psi_{r} X\right), \psi_{r} W\right) \quad \text { for } X \in D, W, Z \in D^{\perp} .
\end{aligned}
$$

Since $M$ is a QR-product the leaf $M^{\perp}$ of $D^{\perp}$ is totally geodesic. Thus using Lemma 2.4 we get (a).

Next for $X \in D, Z \in D^{\perp}$ we have

$$
\bar{\nabla}_{X} \psi_{r} Z=\psi_{r} \bar{\nabla}_{X} Z
$$

which, by virtue of (2.2), gives

$$
\psi_{r} \nabla_{X} Z=-A_{\psi_{r} Z} X+\nabla_{X}^{\perp} \psi_{r} Z-\psi_{r} h(X, Z)
$$

Taking inner products with $Y \in D$ and using the fact that the leaf $M^{\perp}$ of $D^{\perp}$ is totally geodesic, we find

$$
g\left(\psi_{r} \nabla_{X} Z, Y\right)=-g\left(A_{\psi_{r} Z} X, Y\right)=-g\left(h(X, Y), \psi_{r} Z\right) \quad \text { for } X, Y \in D, Z \in D^{\perp} .
$$

On the other hand, for $X \in D$ and $W, Z \in D^{\perp}$ and the use of Lemma 2.5, (3.13) gives

$$
\begin{aligned}
g\left(\psi_{r} \nabla_{X} Z, W\right) & =-g\left(\psi_{r} h(X, Z), W\right)-g\left(h(X, W), \psi_{r} Z\right) \\
& =g\left(A_{\psi_{r} W} Z, X\right)-g\left(A_{\psi_{r} Z} W, X\right) \\
& =g\left(A_{\psi_{r} W} Z-A_{\psi_{r} Z} W, X\right) \\
& =0 .
\end{aligned}
$$

Thus from (3.14) and (3.15) we see that $\psi_{r} \nabla_{X} Z$ is normal to $M$. So $\nabla_{X} Z \in D^{\perp}$ for all $X \in D$ and $Z \in D^{\perp}$.

THEOREM 3.5. Let $M$ be a quaternion CR-submanifold of a quaternion Kaehler manifold $\bar{M}$. Then $M$ is a $Q R$-product if and only if $P_{r}$ is parallel.

Proof. Suppose $P_{r}$ is parallel, then from (3.2), we have

$$
A_{Q_{r} Y} X+t_{r} h(X, Y)=0 \quad \forall X, Y \in T M .
$$

If $Y \in D$, then $Q_{r} Y=0$. Hence (3.16) is reduced to $t_{r} h(X, Y)=0$ for all $X \in T M, Y \in D$. Therefore by virtue of [1, Lemma 5.1, page 403], we get $h\left(D, D^{\perp}\right)=0$ or $h(D, D)=0$. So the quaternion distribution $D$ is integrable by virtue of Lemma 2.3. Thus it follows that each leaf $M^{\perp}$ is totally geodesic in $\bar{M}$ and in particular $M^{\perp}$ is totally geodesic in $M$ by virtue of Lemma 2.2. 
Again from (3.2), we have

$$
A_{\psi_{r} W} Z+t_{r} h(W, Z)=0 \quad \forall W, Z \in D^{\perp} .
$$

So for $X \in D$, we have

$$
g\left(A_{\psi_{r} W} Z, X\right)+g\left(t_{r} h(W, Z), X\right)=0
$$

which means

$$
g\left(h(X, Z), Q_{r} W\right)-g\left(h(W, Z), Q_{r} X\right)=0,
$$

that is,

$$
g\left(h(X, Z), Q_{r} W\right)=0
$$

or

$$
g\left(h\left(D, D^{\perp}\right), Q_{r} D^{\perp}\right)=0 .
$$

Thus using Lemma 2.4, it follows that the leaf $M^{\perp}$ of $D^{\perp}$ is totally geodesic. Hence $M$ is a QR-product.

Conversely, let $M$ be a QR-product. First we show that $\nabla_{U} X \in D$ for any $X \in D$ and $U$ tangent to $M$. Since $M$ is a QR-product, that is, locally a Riemannian product of a quaternion submanifold and a totally real submanifold, it is sufficient to show that $\nabla_{Z} X \in D$ for any $X \in D, Z \in D^{\perp}$ but this was proved in Lemma 3.4(a). Using this fact, we have

$$
\nabla_{U} \psi_{r} X+h\left(U, \psi_{r} X\right)=\psi_{r} \nabla_{U} X+\psi_{r} h(X, U) \text { for any } X \in D, U \text { tangent to } M,
$$

which yields

$$
\psi_{r} h(U, X)=h\left(U, \psi_{r} X\right), \quad \nabla_{U} \psi_{r} X=\psi_{r} \nabla_{U} X .
$$

Thus $\left(\bar{\nabla}_{U} P_{r}\right)(X)=\nabla_{U} P_{r} X-P_{r} \nabla_{U} X=0$, for any $X \in D$, and $U$ tangent to $M$.

Similarly, by using Lemma 3.4(b), it follows that $\nabla_{U} Z \in D^{\perp}$ for any $Z \in D^{\perp}$, and $U$ tangent to $M$. But since $M$ is a QR-product, it follows that $\nabla_{X} Z \in D^{\perp}$ for $U=X \in D$ and $Z \in D^{\perp}$.

Thus, we have $\left(\bar{\nabla}_{U} P_{r}\right)(Z)=0$ for any $Z \in D^{\perp}, U$ tangent to $M$. Therefore $\bar{\nabla} P_{r}=0$, which completes the proof.

COROLLARY 3.6. Let $M$ be a QR-product of a quaternion Kaehler manifold $\bar{M}$. Then $M$ is mixed totally geodesic, that is, $h\left(D, D^{\perp}\right)=0$.

REMARK 3.7. If $M$ is a proper QR-product of a quaternion space form $\bar{M}(c)$, then the ambient manifold $\bar{M}$ is necessarily a space of zero curvature. Hence there does not exist a proper QR-product of a quaternion space form $\bar{M}(c)$ with $c \neq 0$.

\section{Canonical foliations and index of a quaternion CR-submanifold}

DEFINITION 4.1 (see [8]). Let $D$ be a distribution on the Riemannian manifold $M$, $D^{\perp}$ the orthogonal distribution, $\Pi^{\perp}: T M \rightarrow D^{\perp}$ the projection and $\nabla$ the Levi-Civita 
connection. Then the second fundamental form of the plane field $D$, is defined by

$$
S_{\nabla}(X, Y)=\frac{1}{2} \Pi^{\perp}\left(\nabla_{X} Y+\nabla_{Y} X\right) .
$$

The distribution $D$ is called a totally geodesic plane field, if the geodesics tangent to it at one point remain tangent for all their length.

Thus we say that the distribution $D$ is a totally geodesic plane field if

$$
S_{\nabla}(X, Y)=\Pi^{\perp}\left(\nabla_{X} Y+\nabla_{Y} X\right)=0 \quad \forall X, Y \in D .
$$

A geometric definition of this notion is given in [9].

A foliation $f$ on a Riemannian manifold $M$ is called a Riemannian foliation, if the Bott connection $\stackrel{\circ}{\nabla}_{X} Y=\Pi[X, Y]$ in the normal bundle of $f$ preserves the Riemannian metric. Also $f$ is a Riemannian foliation if and only if the second fundamental form $S_{\nabla}$ of the plane field $D$ vanishes (see [9, page 157]).

THEOREM 4.2. Let M be a quaternion CR-submanifold of a quaternion Kaehler manifold $\bar{M}$ such that $D_{M}^{\perp}$ is a totally real foliation of $M$. Then the Bott connection of $D_{M}^{\perp}$ preserves the volume form $\psi$ of $D_{M}$, that is, $\stackrel{\circ}{\nabla}_{Z} \psi=0$, for all $Z \in D_{M}^{\perp}$.

Proof. For any $X, Y \in D$ and $Z \in D^{\perp}$, we have

$$
\begin{aligned}
g\left(\left(\stackrel{\circ}{\nabla}_{Z} \psi_{r}\right)(X), Y\right)= & g\left(\stackrel{\circ}{\nabla}_{Z} \psi_{r} X, Y\right)-g\left(\psi_{r} \stackrel{\circ}{\nabla}_{Z} X, Y\right) \\
= & g\left(\left[Z, \psi_{r} X\right], Y\right)+g\left([Z, X], \psi_{r} Y\right) \\
= & g\left(\bar{\nabla}_{Z} \psi_{r} X, Y\right)-g\left(\bar{\nabla}_{\psi_{r} X} Z, Y\right) \\
& +g\left(\bar{\nabla}_{Z} X, \psi_{r} Y\right)-g\left(\bar{\nabla}_{X} Z, \psi_{r} Y\right) \\
= & g\left(X, \psi_{r} \bar{\nabla}_{Z} Y\right)+g\left(\bar{\nabla}_{\psi_{r} X} Y, Z\right) \\
& -g\left(X, \bar{\nabla}_{Z} \psi_{r} Y\right)+g\left(\bar{\nabla}_{X} \psi_{r} Y, Z\right) \\
= & g\left(\bar{\nabla}_{\psi_{r} X} Y, Z\right)+g\left(\bar{\nabla}_{X} \psi_{r} Y, Z\right) \\
= & g\left(\bar{\nabla}_{\psi_{r} X} Y, Z\right)+g\left(\bar{\nabla}_{X} \psi_{r} Z, Y\right) \\
= & g\left(\bar{\nabla}_{\psi_{r} X} Y, Z\right)-g\left(A_{\psi_{r} Z} X, Y\right) .
\end{aligned}
$$

Also,

$$
\begin{aligned}
g\left(\nabla_{X} X, Z\right) & =g\left(\bar{\nabla}_{X} X, Z\right) \\
& =g\left(\psi_{r} \bar{\nabla}_{X} X, \psi_{r} Z\right) \\
& =g\left(\bar{\nabla}_{X} \psi_{r} X, \psi_{r} Z\right) \\
& =-g\left(\bar{\nabla}_{X} \psi_{r} Z, \psi_{r} X\right) \\
& =g\left(A_{\psi_{r} Z} X, \psi_{r} X\right) .
\end{aligned}
$$

If $D_{M}^{\perp}$ is Riemannian then $D_{M}$ is a totally geodesic plane field and so (4.4) gives $g\left(A_{\psi_{r} Z} X, \psi_{r} X\right)=0$. 
Therefore $g\left(A_{\psi_{r} Z}(X+Y), \psi_{r}(X+Y)\right)=0$, and hence we obtain

$$
g\left(A_{\psi_{r} Z} X, \psi_{r} Y\right)+g\left(A_{\psi_{r} Z} Y, \psi_{r} X\right)=0 .
$$

Thus using (4.3) and (4.5), we have

$$
\begin{aligned}
g\left(\left(\stackrel{\circ}{\nabla}_{Z} \psi_{r}\right)(X), \psi_{r} Y\right) & =g\left(\bar{\nabla}_{\psi_{r} X} \psi_{r} Y, Z\right)-g\left(A_{\psi_{r} Z} X, \psi_{r} Y\right) \\
& =g\left(\bar{\nabla}_{\psi_{r} X} \psi_{r} Y, Z\right)+g\left(A_{\psi_{r} Z} Y, \psi_{r} X\right) \\
& =0 .
\end{aligned}
$$

Moreover, it is known that $D_{M}$ is a minimal distribution [2], which implies that

$$
(d \psi)\left(Z, X_{1}, \ldots, X_{4 n}\right)=0 \text { for } Z \in D^{\perp}, X_{1}, \ldots, X_{4 n} \in D .
$$

Hence

$$
\begin{aligned}
\left(\stackrel{\circ}{\nabla}_{Z} \psi\right)\left(X_{1}, \ldots, X_{4 n}\right) & =Z \psi\left(X_{1}, \ldots, X_{4 n}\right)-\sum_{a=1}^{4 n} \psi\left(X_{1}, \ldots, \Pi\left[Z, X_{a}\right], \ldots, X_{4 n}\right) \\
& =(d \psi)\left(Z, X_{1}, \ldots, X_{4 n}\right)=0,
\end{aligned}
$$

which completes the proof.

Now, let $M$ be a compact totally geodesic quaternion CR-submanifold of a quaternion Kaehler manifold $\bar{M}$. Let $N$ be a normal vector field and denote by $v^{\prime \prime}(N)$ the second normal variation of $M$ induced by $N$. Then we have (see [6, Chapter 1]),

$$
v^{\prime \prime}(N)=\int_{M}\left\{\left\|\nabla^{\perp} N\right\|^{2}-\sum_{i=1}^{n} \bar{R}\left(X_{i}, N, N, X_{i}\right)-\left\|A_{N}\right\|^{2}\right\} d V,
$$

where $N \in T^{\perp} M, d V$ denotes the volume element of $M$ and $\left\{X_{i}\right\}$ is an orthonormal frame in $T M$. Applying the Stokes theorem to the integral of the first term of (4.9), we have

$$
I(N, N)=: v^{\prime \prime}(N)=\int_{M} g(L N, N) * 1,
$$

where $L$ is a selfadjoint, strongly elliptic linear differential operator of the second order. The differential operator $L$ is called the Jacobi operator of $M$ in $\bar{M}$ and has discrete eigenvalues $\lambda_{1}<\lambda_{2}<\cdots$. We put $E_{\lambda}=\left\{N \in T^{\perp} M: L(N)=\lambda N\right\}$. The dimension of the space $E_{\lambda}, \operatorname{dim}\left(E_{\lambda}\right)$, is called the index of $M$ in $\bar{M}$. For two normal vector fields $N_{1}, N_{2}$ to a minimal submanifold $M$ in $\bar{M}$, their index form is defined by

$$
I\left(N_{1}, N_{2}\right)=\int_{M} g\left(L N_{1}, N_{2}\right) * 1 .
$$

It is easy to see that the index form $I$ is a symmetric bilinear form; $I: T^{\perp} M \times T^{\perp} M \rightarrow R$.

Now we prove the following theorem.

THEOREM 4.3. Let $M$ be a compact n-dimensional minimal quaternion CR-submanifold of a quaternion Kaehler manifold $\bar{M}$. If $M$ has nonpositive holomorphic bisectional curvature, then the index form satisfies

$$
I(N, N)+I\left(\psi_{r} N, \psi_{r} N\right) \geq 0 \quad \text { for any } N \in \mu .
$$


Proof. By using the Weingarten equation we have that for all $X, Y \in D^{\perp}$,

$$
\begin{aligned}
g\left(\nabla_{X}^{\perp} N, \psi_{r} Y\right) & =g\left(\bar{\nabla}_{X} N, \psi_{r} Y\right) \\
& =-g\left(\psi_{r} \bar{\nabla}_{X} N, Y\right) \\
& =-g\left(\bar{\nabla}_{X} \psi_{r} N, Y\right) \\
& =g\left(A_{\psi_{r} N} X, Y\right)
\end{aligned}
$$

which implies that

$$
\left\|\nabla^{\perp} N\right\|^{2} \geq\left\|A_{\psi_{r} N}\right\|^{2}, \quad\left\|\nabla^{\perp} \psi_{r} N\right\|^{2} \geq\left\|A_{N}\right\|^{2} \quad \text { for any } N \in \mu,
$$

where $\mu$ is defined in (2.4). Thus by using (4.9), (4.10), (4.13), and (4.14) we get

$$
I(N, N)+I\left(\psi_{r} N, \psi_{r} N\right) \geq-\int_{M} \sum_{i=1}^{n}\left\{\bar{R}\left(N, e_{i}, e_{i}, N\right)+\bar{R}\left(\psi_{r} N, e_{i}, e_{i}, \psi_{r} N\right)\right\} * 1
$$

from which the proof follows, since $M$ has nonpositive holomorphic bisectional curvature.

Finally, we prove a classification theorem for the totally umbilical quaternion CRsubmanifolds of a quaternion Kaehler manifold.

THEOREM 4.4. Let $M$ be a compact totally umbilical quaternion CR-submanifold of a quaternion Kaehler manifold $\bar{M}$. Then

(a) $M$ is a totally geodesic submanifold, or,

(b) $M$ is locally the Riemannian product of a quaternion submanifold and a totally real submanifold, or,

(c) $M$ is a totally real submanifold, or,

(d) the totally real distribution is one dimensional, that is, $\operatorname{dim} D^{\perp}=1$,

(e) $\nabla_{X}^{\perp} H \in \mu$, for $X \in D$.

Proof. We take $X, W \in D^{\perp}$ and using (2.6) with the fact that $\bar{M}$ is a quaternion Kaehler manifold, we have

$$
\psi_{r} \nabla_{X} W+g(X, W) \psi_{r} H=-A_{\psi_{r} W} X+\nabla_{X}^{\frac{1}{X}} \psi_{r} W
$$

Taking inner product with $X$ we get

$$
g\left(H, \psi_{r} W\right)\|X\|^{2}=g(X, W) g\left(H, \psi_{r} X\right) .
$$

Exchanging $X$ and $W$ in (4.17) we have

$$
\mathcal{g}\left(H, \psi_{r} X\right)\|W\|^{2}=\mathfrak{g}(X, W) \mathcal{g}\left(H, \psi_{r} W\right) .
$$

This together with (4.17) gives

$$
\mathcal{g}\left(H, \psi_{r} W\right)=\frac{g(X, W)^{2}}{\|X\|^{2}\|W\|^{2}} g\left(H, \psi_{r} W\right) .
$$


The possible solutions of (4.19) are

(i) $H=0$,

(ii) $H \perp \psi_{r} W$,

(iii) $X \| W$.

Suppose that condition (i) holds, that is, $H=0$. This implies that $M$ is totally geodesic which proves (a). Combining (ii) with a result in [1, page 407] we get (b) of the theorem.

Now from (2.7) we have

$$
\begin{aligned}
O & =\bar{R}(I X, J X, K X, N) \\
& =\bar{R}(K X, N, I X, J X) \\
& =-\bar{R}(K X, N, X, K X) \\
& =-\bar{R}(X, K X, K X, N) \\
& =-g\left(\nabla \frac{1}{X} H, N\right)\|X\|^{2}
\end{aligned}
$$

which implies that

$$
\nabla_{X}^{\perp} H \in \mu \quad \forall X \in D
$$

proving (e). Next we have

$$
\bar{\nabla}_{X} \psi_{r} H=\psi_{r} \bar{\nabla}_{X} H \quad \text { for } X \in D
$$

which, by (2.6) gives

$$
\nabla_{X}^{\perp} \psi_{r} H=-g(H, H) \psi_{r} X+\psi_{r} \nabla_{\bar{X}}^{\perp} H .
$$

Since $\nabla_{X}^{\frac{1}{X}} H \in \mu$, from (4.23) we have $\psi_{r} X=0$ for all $X \in D$. Hence $D=\{0\}$ which proves (c). Finally if (iii) is valid then $\operatorname{dim} D^{\perp}=1$, which completes the proof.

ACKNOWLEDGEMENTS. The authors would like to acknowledge the financial support of the Research Committee, University of Patras, Programme Karatheodori (\# 2461), and of the Greek State Scholarships Foundation (I.K.Y).

\section{REFERENCES}

[1] M. Barros, B. Chen, and F. Urbano, Quaternion CR-submanifolds of quaternion manifolds, Kodai Math. J. 4 (1981), no. 3, 399-417. MR 83e:53055. Zbl 481.53046.

[2] M. Barros and F. Urbano, Topology of quaternion CR-submanifolds, Boll. Un. Mat. Ital. A (6) 2 (1983), no. 1, 103-110. MR 84h:53071. Zbl 518.53054.

[3] A. Bejancu, CR-submanifolds of a Kaehler manifold. I, Proc. Amer. Math. Soc. 69 (1978), no. 1, 135-142. MR 57\#7486. Zbl 368.53040.

[4] _ Geometry of CR-submanifolds, Mathematics and its Applications (East European Series), vol. 23, D. Reidel, Dordrecht, 1986. MR 87k:53126. Zbl 605.53001.

[5] B. Chen, CR-submanifolds of a Kaehler manifold. I, J. Differential Geom. 16 (1981), no. 2, 305-322. MR 84e:53062a. Zbl 431.53048.

[6] __ Geometry of Submanifolds and its Applications, Science University of Tokyo, Tokyo, 1981. MR 82m:53051. Zbl 474.53050.

[7] S. Ishihara, Quaternion Kaehlerian manifolds, J. Differential Geom. 9 (1974), 483-500. Zbl 297.53014.

[8] B. L. Reinhart, The second fundamental form of a plane field, J. Differential Geom. 12 (1977), no. 4, 619-627. MR 80a:57013. Zbl 379.53018. 
[9]__ Differential Geometry of Foliations. The Fundamental Integrability Problem, Ergebnisse der Mathematik und ihrer Grenzgebiete [Results in Mathematics and Related Areas], vol. 99, Springer-Verlag, Berlin, 1983. MR 85i:53038. Zbl 506.53018.

Bassil J. Papantoniou: Department of Mathematics, University of Patras, 26100, PATRAS, GREECE

E-mail address: bipapant@math.upatras.gr

M. Hasan Shahid: Department of Mathematics, Faculty of Natural Science, Jamia Millia ISLAMia, New DelHI, INDiA

E-mail address: hasan.mt@jmi .ernet.in 


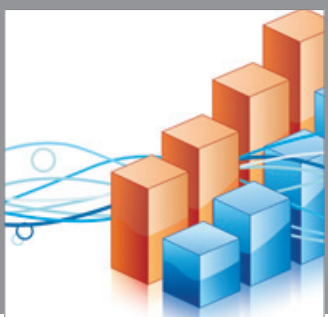

Advances in

Operations Research

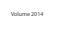

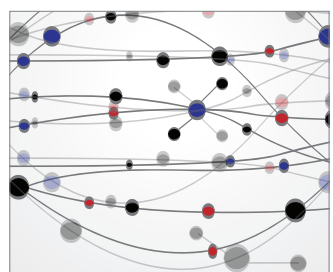

\section{The Scientific} World Journal
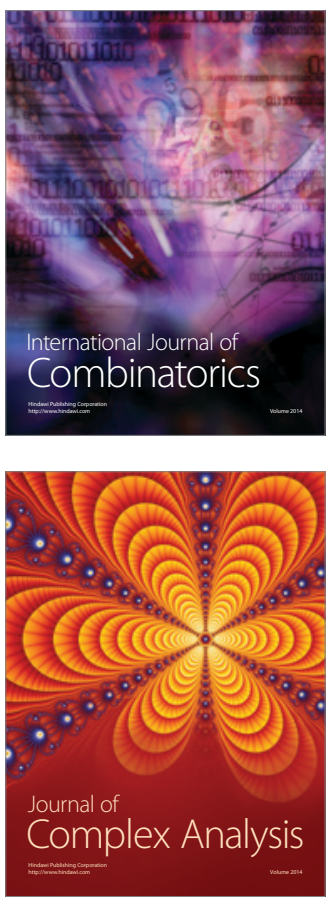

International Journal of

Mathematics and

Mathematical

Sciences
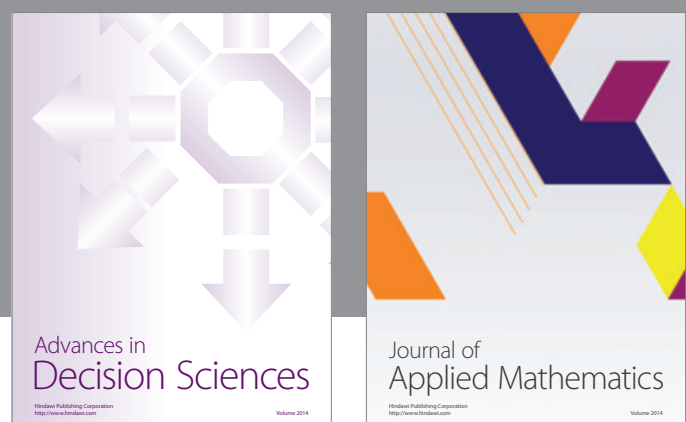

Journal of

Applied Mathematics
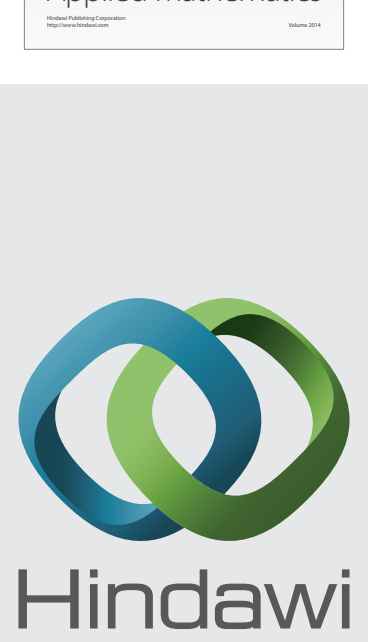

Submit your manuscripts at http://www.hindawi.com
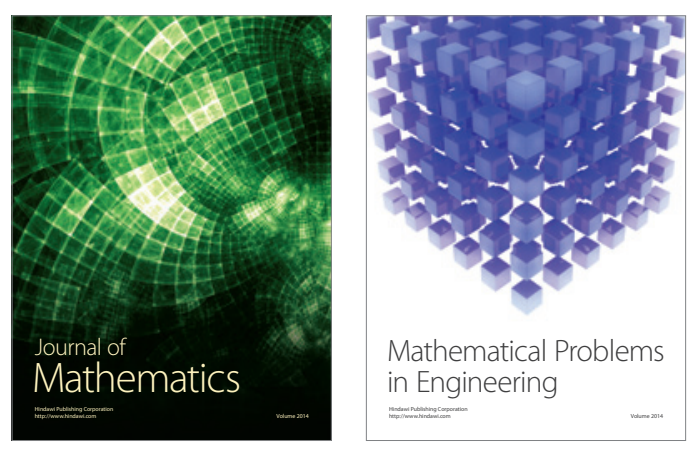

Mathematical Problems in Engineering
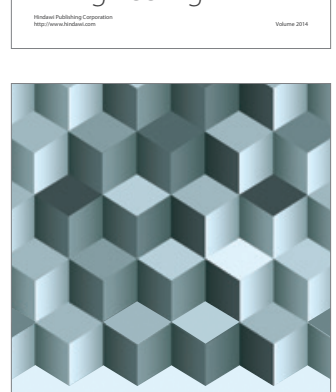

Journal of

Function Spaces
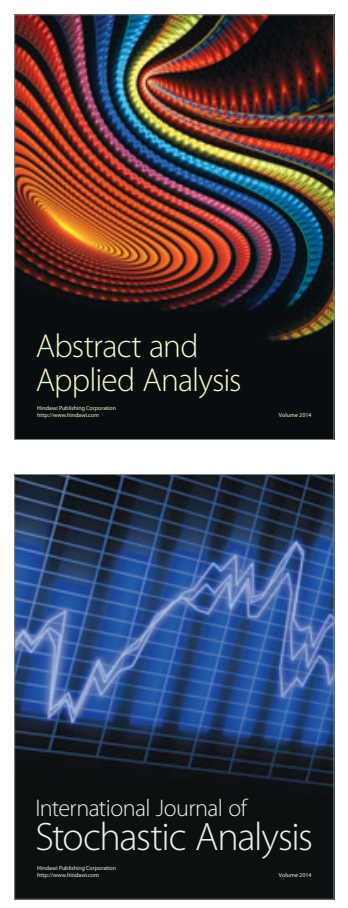

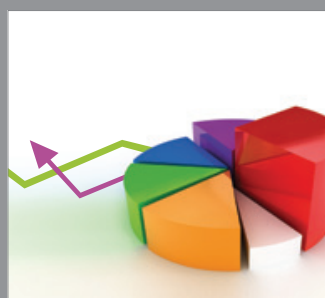

ournal of

Probability and Statistics

Promensencen
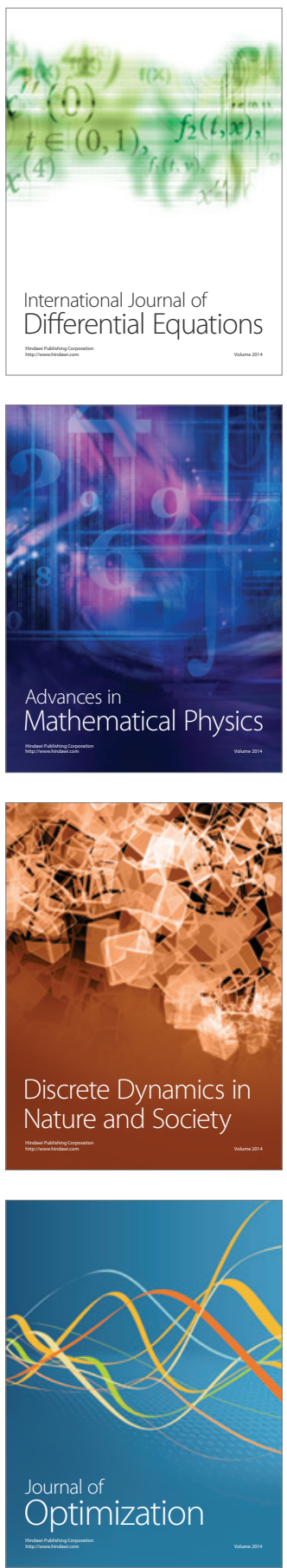